\title{
WEIGHTED INTEGRAL MEANS OF MIXED AREAS AND LENGTHS UNDER HOLOMORPHIC MAPPINGS
}

\author{
JIE XIAO AND WEN XU
}

\begin{abstract}
Aвstract. This note addresses monotonic growths and logarithmic convexities of the weighted $\left(\left(1-t^{2}\right)^{\alpha} d t^{2},-\infty<\alpha<\infty, 0<t<1\right)$ integral means $\mathrm{A}_{\alpha, \beta}(f, \cdot)$ and $\mathrm{L}_{\alpha, \beta}(f, \cdot)$ of the mixed area $\left(\pi r^{2}\right)^{-\beta} A(f, r)$ and the mixed length $(2 \pi r)^{-\beta} L(f, r)$ $(0 \leq \beta \leq 1$ and $0<r<1)$ of $f(r \mathbb{D})$ and $\partial f(r \mathbb{D})$ under a holomorphic map $f$ from the unit disk $\mathbb{D}$ into the finite complex plane $\mathbb{C}$.
\end{abstract}

\section{INTRODUCTION}

From now on, $\mathbb{D}$ represents the unit disk in the finite complex plane $\mathbb{C}, H(\mathbb{D})$ denotes the space of holomorphic mappings $f: \mathbb{D} \rightarrow \mathbb{C}$, and $U(\mathbb{D})$ stands for all univalent functions in $H(\mathbb{D})$. For any real number $\alpha$, positive number $r \in(0,1)$ and the standard area measure $d A$, let

$$
d A_{\alpha}(z)=\left(1-|z|^{2}\right)^{\alpha} d A(z) ; \quad r \mathbb{D}=\{z \in \mathbb{D}:|z|<r\} ; \quad r \mathbb{T}=\{z \in \mathbb{D}:|z|=r\} .
$$

In their recent paper [11], Xiao and Zhu have discussed the following area $0<$ $p<\infty$-integral means of $f \in H(\mathbb{D})$ :

$$
M_{p, \alpha}(f, r)=\left[\frac{1}{A_{\alpha}(r \mathbb{D})} \int_{r \mathbb{D}}|f|^{p} d A_{\alpha}\right]^{\frac{1}{p}},
$$

proving that $r \mapsto M_{p, \alpha}(f, r)$ is strictly increasing unless $f$ is a constant, and $\log r \mapsto$ $\log M_{p, \alpha}(f, r)$ is not always convex. This last result suggests a conjecture that $\log r \mapsto \log M_{p, \alpha}(f, r)$ is convex or concave when $\alpha \leq 0$ or $\alpha>0$. But, motivated by [11, Example 10, (ii)] we can choose $p=2, \alpha=1, f(z)=z+c$ and $c>0$ to verify that the conjecture is not true. At the same time, this negative result was also obtained in Wang-Zhu's manuscript [10]. So far it is unknown whether the conjecture is generally true for $p \neq 2$.

The foregoing observation has actually inspired the following investigation. Our concentration is the fundamental case $p=1$. To understand this approach, let us take a look at $M_{1, \alpha}(\cdot, \cdot)$ from a differential geometric viewpoint. Note that

$$
M_{1, \alpha}\left(f^{\prime}, r\right)=\frac{\int_{r \mathbb{D}}\left|f^{\prime}\right| d A_{\alpha}}{A_{\alpha}(r \mathbb{D})}=\frac{\int_{0}^{r}\left[(2 \pi t)^{-1} \int_{t \mathbb{T}}\left|f^{\prime}(z)\right||d z|\right]\left(1-t^{2}\right)^{\alpha} d t^{2}}{\int_{0}^{r}\left(1-t^{2}\right)^{\alpha} d t^{2}} .
$$

2000 Mathematics Subject Classification. 32A10, 32A36, 51M25.

Key words and phrases. monotonic growth, logarithmic convexity, mean mixed area, mean mixed length, isoperimetric inequality, holomorphic map, univalent function.

JX and WX were in part supported by NSERC of Canada and the Finnish Cultural Foundation, respectively. 
So, if $f \in U(\mathbb{D})$, then

$$
(2 \pi t)^{-1} \int_{t \mathbb{T}}\left|f^{\prime}(z)\right||d z|
$$

is a kind of mean of the length of $\partial f(t \mathbb{D})$, and hence the square of this mean dominates a sort of mean of the area of $f(t \mathbb{D})$ in the isoperimetric sense:

$$
\Phi_{A}(f, t)=\left(\pi t^{2}\right)^{-1} \int_{t \mathbb{D}}\left|f^{\prime}(z)\right|^{2} d A(z) \leq\left[(2 \pi t)^{-1} \int_{t \mathbb{T}}\left|f^{\prime}(z)\right||d z|\right]^{2}=\left[\Phi_{L}(f, t)\right]^{2} .
$$

According to the Pólya-Szegö monotone principle [9, Problem 309] (or [3, Proposition 6.1]) and the area Schwarz's lemma in Burckel, Marshall, Minda, PoggiCorradini and Ransford [3, Theorem 1.9], $\Phi_{L}(f, \cdot)$ and $\Phi_{A}(f, \cdot)$ are strictly increasing on $(0,1)$ unless $f(z)=a_{1} z$ with $a_{1} \neq 0$. Furthermore, $\log \Phi_{L}(f, r)$ and $\log \Phi_{A}(f, r)$, equivalently, $\log L(f, r)$ and $\log A(f, r)$, are convex functions of $\log r$ for $r \in(0,1)$, due to the classical Hardy's convexity and [3. Section 5]. Perhaps, it is worth-wise to mention that if $c>0$ is small enough then the universal cover of $\mathbb{D}$ onto the annulus $\left\{e^{-\frac{c \pi}{2}}<|z|<e^{\frac{c \pi}{2}}\right\}$ :

$$
f(z)=\exp \left[i c \log \left(\frac{1+z}{1-z}\right)\right]
$$

enjoys the property that $\log r \mapsto \log A(f, r)$ is not convex; see [3, Example 5.1].

In the above and below, we have used the following convention:

$$
\Phi_{A}(f, r)=\frac{A(f, r)}{\pi r^{2}} \quad \& \quad \Phi_{L}(f, r)=\frac{L(f, r)}{2 \pi r},
$$

where under $r \in(0,1)$ and $f \in H(\mathbb{D}), A(f, r)$ and $L(f, r)$ stand respectively for the area of $f(r \mathbb{D})$ (the projection of the Riemannian image of $r \mathbb{D}$ by $f$ ) and the length of $\partial f(r \mathbb{D})$ (the boundary of the projection of the Riemannian image of $r \mathbb{D}$ by $f$ ) with respect to the standard Euclidean metric on $\mathbb{C}$. For our purpose, we choose a shortcut notation

$$
d \mu_{\alpha}(t)=\left(1-t^{2}\right)^{\alpha} d t^{2} \quad \& \quad v_{\alpha}(t)=\mu_{\alpha}([0, t]) \quad \forall \quad t \in(0,1),
$$

and for $0 \leq \beta \leq 1$ define

$$
\Phi_{A, \beta}(f, t)=\frac{A(f, t)}{\left(\pi t^{2}\right)^{\beta}} \quad \& \quad \Phi_{L, \beta}(f, t)=\frac{L(f, t)}{(2 \pi t)^{\beta}},
$$

and then

$$
\mathrm{A}_{\alpha, \beta}(f, r)=\frac{\int_{0}^{r} \Phi_{A, \beta}(f, t) d \mu_{\alpha}(t)}{\int_{0}^{r} d \mu_{\alpha}(t)} \quad \& \quad \mathrm{~L}_{\alpha, \beta}(f, r)=\frac{\int_{0}^{r} \Phi_{L, \beta}(f, t) d \mu_{\alpha}(t)}{\int_{0}^{r} d \mu_{\alpha}(t)}
$$

which are called the weighted integral means of the mixed area and the mixed length for $f(r \mathbb{D})$ and $\partial f(r \mathbb{D})$, respectively.

In this note, we consider two fundamental properties: monotonic growths and logarithmic convexities of both $\mathrm{A}_{\alpha, \beta}(f, r)$ and $\mathrm{L}_{\alpha, \beta}(f, r)$, thereby producing two 
specialities: (i) if $r \mapsto \Phi_{L}(f, r)$ is monotone increasing on $(0,1)$, then so is the isoperimetry-induced function:

$$
r \mapsto \frac{\int_{0}^{r}\left[\Phi_{L, 1}(f, t)\right]^{2} d \mu_{\alpha}(t)}{\int_{0}^{r} d \mu_{\alpha}(t)} \geq \mathrm{A}_{\alpha, 1}(f, r) ;
$$

(ii) the log-convexity for $\mathrm{L}_{\alpha, \beta=1}(f, r)$ essentially settles the above-mentioned conjecture. The details (results and their proofs) are arranged in the forthcoming two sections.

\section{Monotonic Growth}

In this section, we deal with the monotonic growths of $\mathrm{A}_{\alpha, \beta}(f, r)$ and $\mathrm{L}_{\alpha, \beta}(f, r)$, along with their associated Schwarz type lemmas. In what follows, $\mathbb{N}$ is used as the set of all natural numbers.

2.1. Two Lemmas. The following two preliminary results are needed.

Lemma 1. [6, Theorems 1 \& 2] Let $f \in H(\mathbb{D})$ be of the form $f(z)=a_{0}+\sum_{k=n}^{\infty} a_{k} z^{k}$ with $n \in \mathbb{N}$. Then:

(i) $\pi r^{2 n}\left[\frac{\left|f^{(n)}(0)\right|}{n !}\right]^{2} \leq A(f, r) \quad \forall \quad r \in(0,1)$

(ii) $2 \pi r^{n}\left[\frac{\left|f^{(n)}(0)\right|}{n !}\right] \leq L(f, r) \quad \forall \quad r \in(0,1)$.

Moreover, equality in (i) or (ii) holds if and only if $f(z)=a_{0}+a_{n} z^{n}$.

Proof. This may be viewed as the higher order Schwarz type lemma for area and length. See also the proofs of Theorems $1 \& 2$ in [6], and their immediate remarks on equalities. Here it is worth noticing three matters: (a) $\frac{f^{(n)}(0)}{n !}$ is just $a_{n}$; (b) [5, Corollary 3] presents a different argument for the area case; (c) $L(f, r)$ is greater than or equal to the length $l(r, f)$ of the outer boundary of $f(r \mathbb{D})$ (defined in [6]) which is not less than the length $l^{\#}(r, f)$ of the exact outer boundary of $f(r \mathbb{D})$ (introduced in [12]).

Lemma 2. Let $0 \leq \beta \leq 1$.

(i) If $f \in H(\mathbb{D})$, then $r \mapsto \Phi_{A, \beta}(f, r)$ is strictly increasing on $(0,1)$ unless

$$
f=\left\{\begin{aligned}
\text { constant } & \text { when } \quad \beta<1 \\
\text { linear map } & \text { when } \quad \beta=1 .
\end{aligned}\right.
$$

(ii) If $f \in U(\mathbb{D})$ or $f(z)=a_{0}+a_{n} z^{n}$ with $n \in \mathbb{N}$, then $r \mapsto \Phi_{L, \beta}(f, r)$ is strictly increasing on $(0,1)$ unless

$$
f=\left\{\begin{aligned}
\text { constant } & \text { when } \quad \beta<1 \\
\text { linear map } & \text { when } \quad \beta=1 .
\end{aligned}\right.
$$

Proof. It is enough to handle $\beta<1$ since the case $\beta=1$ has been treated in [3, Theorem $1.9 \&$ Proposition 6.1]. The monotonic growths in (i) and (ii) follow from

$$
\Phi_{A, \beta}(f, r)=\left(\pi r^{2}\right)^{1-\beta} \Phi_{A, 1}(f, r) \quad \& \quad L(f, r)=(2 \pi r)^{1-\beta} \Phi_{L, 1}(f, r) .
$$

To see the strictness, we consider two cases. 
(i) Suppose that $\Phi_{A, \beta}(f, \cdot)$ is not strictly increasing. Then there are $r_{1}, r_{2} \in(0,1)$ such that $r_{1}<r_{2}$, and $\Phi_{A, \beta}(f, \cdot)$ is a constant on $\left[r_{1}, r_{2}\right]$. Hence

$$
\frac{d}{d r} \Phi_{A, \beta}(f, r)=0 \quad \forall \quad r \in\left[r_{1}, r_{2}\right] .
$$

Equivalently,

$$
2 \beta A(f, r)=r \frac{d}{d r} A(f, r) \quad \forall \quad r \in\left[r_{1}, r_{2}\right] .
$$

But, according to [3, (4.2)]:

$$
2 A(f, r) \leq r \frac{d}{d r} A(f, r) \quad \forall \quad r \in(0,1) .
$$

Since $\beta<1$, we get $A(f, r)=0$ for all $r \in\left[r_{1}, r_{2}\right]$, whence finding that $f$ is constant.

(ii) Now assume that $\Phi_{L, \beta}(f, \cdot)$ is not strictly increasing. There are $r_{3}, r_{4} \in(0,1)$ such that $r_{3}<r_{4}$ and

$$
0=\frac{d}{d r} \Phi_{L, \beta}(f, r)=(2 \pi r)^{-\beta}\left[\frac{d}{d r} L(f, r)-\frac{\beta}{r} L(f, r)\right]=0 \quad \forall \quad r \in\left[r_{3}, r_{4}\right] .
$$

If $f \in U(\mathbb{D})$ then

$$
L(f, r)=\int_{r \mathbb{T}}\left|f^{\prime}(z)\right||d z|
$$

and hence one has the following "first variation formula"

$$
\frac{d}{d r} L(f, r)=\int_{0}^{2 \pi}\left|f^{\prime}\left(r e^{i \theta}\right)\right| d \theta+r \frac{d}{d r} \int_{0}^{2 \pi}\left|f^{\prime}\left(r e^{i \theta}\right)\right| d \theta \quad \forall \quad r \in\left[r_{3}, r_{4}\right] .
$$

The previous three equations yield

$$
0=(1-\beta) \int_{0}^{2 \pi}\left|f^{\prime}\left(r e^{i \theta}\right)\right| d \theta+r \frac{d}{d r} \int_{0}^{2 \pi}\left|f^{\prime}\left(r e^{i \theta}\right)\right| d \theta \quad \forall \quad r \in\left[r_{3}, r_{4}\right]
$$

and so

$$
\int_{0}^{2 \pi}\left|f^{\prime}\left(r e^{i \theta}\right)\right| d \theta=0 \quad \forall \quad r \in\left[r_{3}, r_{4}\right] .
$$

This ensures that $f$ is a constant, contradicting $f \in U(\mathbb{D})$. Therefore, $f(z)$ is of the form $a_{0}+a_{n} z^{n}$. But, since $L\left(z^{n}, r\right)=2 \pi r^{n}$ is strictly increasing, $f$ must be constant.

2.2. Monotonic Growth of $\mathbf{A}_{\alpha, \beta}(f, \cdot)$. This aspect is essentially motivated by the following Schwarz type lemma.

Proposition 1. Let $-\infty<\alpha<\infty, 0 \leq \beta \leq 1$, and $f \in H(\mathbb{D})$ be of the form $f(z)=a_{0}+\sum_{k=n}^{\infty} a_{k} z^{k}$ with $n \in \mathbb{N}$. Then

$$
\pi^{1-\beta}\left[\frac{\left|f^{(n)}(0)\right|}{n !}\right]^{2} \leq \mathrm{A}_{\alpha, \beta}(f, r)\left[\frac{v_{\alpha}(r)}{\int_{0}^{r} t^{2(n-\beta)} d \mu_{\alpha}(t)}\right] \quad \forall \quad r \in(0,1)
$$

with equality if and only if $f(z)=a_{0}+a_{n} z^{n}$. 
Proof. The inequality follows from Lemma 1 (i) right away. When $f(z)=a_{0}+$ $a_{n} z^{n}$, the last inequality becomes equality due to the equality case of Lemma 1 (i). Conversely, suppose that the last inequality is an equality. If $f$ does not have the form $a_{0}+a_{n} z^{n}$, then the equality in Lemma 1 (i) is not true, then there are $r_{1}, r_{2} \in(0,1)$ such that $r_{1}<r_{2}$ and

$$
A(f, t)>\pi t^{2 n}\left[\frac{\left|f^{(n)}(0)\right|}{n !}\right]^{2} \quad \forall \quad t \in\left[r_{1}, r_{2}\right] .
$$

This strict inequality forces that for $r \in\left[r_{1}, r_{2}\right]$,

$$
\begin{aligned}
\pi^{1-\beta}\left[\frac{\left|f^{(n)}(0)\right|}{n !}\right]^{2} \int_{0}^{r} t^{2(n-\beta)} d \mu_{\alpha}(t) & =\int_{0}^{r}\left(\pi t^{2}\right)^{-\beta} A(f, t) d \mu_{\alpha}(t) \\
& =\left(\int_{0}^{r_{1}}+\int_{r_{1}}^{r_{2}}+\int_{r_{2}}^{r}\left(\pi t^{2}\right)^{-\beta} A(f, t) d \mu_{\alpha}(t)\right. \\
& >\pi^{1-\beta}\left[\frac{\left|f^{(n)}(0)\right|}{n !}\right]^{2} \int_{0}^{r} t^{2(n-\beta)} d \mu_{\alpha}(t),
\end{aligned}
$$

a contradiction. Thus $f(z)=a_{0}+a_{n} z^{n}$.

Based on Proposition 1 we find the monotonic growth for $\mathrm{A}_{\alpha, \beta}(\cdot, \cdot)$ as follows.

Theorem 1. Let $-\infty<\alpha<\infty, 0 \leq \beta \leq 1$, and $f \in H(\mathbb{D})$. Then $r \mapsto \mathrm{A}_{\alpha, \beta}(f, r)$ is strictly increasing on $(0,1)$ unless

$$
f=\left\{\begin{aligned}
\text { constant } & \text { when } \quad \beta<1 \\
\text { linear map } & \text { when } \quad \beta=1 .
\end{aligned}\right.
$$

Consequently,

(i)

(ii) If

$$
\lim _{r \rightarrow 0} \mathrm{~A}_{\alpha, \beta}(f, r)=\left\{\begin{array}{r}
0 \\
\left|f^{\prime}(0)\right|^{2} \quad \text { when } \quad \beta<1 \\
\quad \text { when }
\end{array}\right.
$$

$$
\Phi_{A, \beta}(f, 0):=\lim _{r \rightarrow 0} \Phi_{A, \beta}(f, r) \quad \& \quad \Phi_{A, \beta}(f, 1):=\lim _{r \rightarrow 1} \Phi_{A, \beta}(f, r)<\infty,
$$

then

$$
0<r<s<1 \Rightarrow 0 \leq \frac{\mathrm{A}_{\alpha, \beta}(f, s)-\mathrm{A}_{\alpha, \beta}(f, r)}{\log v_{\alpha}(s)-\log v_{\alpha}(r)} \leq \Phi_{A, \beta}(f, s)-\Phi_{A, \beta}(f, 0)
$$

with equality if and only if

$$
f=\left\{\begin{aligned}
\text { constant } & \text { when } \quad \beta<1 \\
\text { linear map } & \text { when } \quad \beta=1 .
\end{aligned}\right.
$$

In particular, $t \mapsto \mathrm{A}_{\alpha, \beta}(f, t)$ is Lipschitz with respect to $\log v_{\alpha}(t)$ for $t \in(0,1)$.

Proof. Note that $v_{\alpha}(r)=\int_{0}^{r} d \mu_{\alpha}(t)$. So $d v_{\alpha}(r)$, the differential of $v_{\alpha}(r)$ with respect to $r \in(0,1)$, equals $d \mu_{\alpha}(r)$. By integration by parts we have

$$
\Phi_{A, \beta}(f, r) v_{\alpha}(r)-\int_{0}^{r} \Phi_{A, \beta}(f, t) d \mu_{\alpha}(t)=\int_{0}^{r}\left[\frac{d}{d t} \Phi_{A, \beta}(f, t)\right] v_{\alpha}(t) d t .
$$


Differentiating the function $\mathrm{A}_{\alpha, \beta}(f, r)$ with respect to $r$ and using Lemma2(i), we get

$$
\begin{aligned}
\frac{d}{d r} \mathrm{~A}_{\alpha, \beta}(f, r) & =\frac{\Phi_{A, \beta}(f, r) 2 r\left(1-r^{2}\right)^{\alpha} v_{\alpha}(r)-\left[\int_{0}^{r} \Phi_{A, \beta}(f, t) d \mu_{\alpha}(t)\right] 2 r\left(1-r^{2}\right)^{\alpha}}{v_{\alpha}(r)^{2}} \\
& =\frac{2 r\left(1-r^{2}\right)^{\alpha}\left[\Phi_{A, \beta}(f, t) v_{\alpha}(r)-\int_{0}^{r} \Phi_{A, \beta}(f, t) d \mu_{\alpha}(t)\right]}{v_{\alpha}(r)^{2}} \\
& =\frac{2 r\left(1-r^{2}\right)^{\alpha} \int_{0}^{r}\left[\frac{d}{d t} \Phi_{A, \beta}(f, t)\right] v_{\alpha}(t) d t}{v_{\alpha}(r)^{2}} \geq 0 .
\end{aligned}
$$

As a result, $r \mapsto \mathrm{A}_{\alpha, \beta}(f, r)$ increases on $(0,1)$.

Next suppose that the just-verified monotonicity is not strict. Then there exist two numbers $r_{1}, r_{2} \in(0,1)$ such that $r_{1}<r_{2}$ and

$$
\mathrm{A}_{\alpha, \beta}\left(f, r_{1}\right)=\mathrm{A}_{\alpha, \beta}(f, r)=\mathrm{A}_{\alpha, \beta}\left(f, r_{2}\right) \quad \forall \quad r \in\left[r_{1}, r_{2}\right] .
$$

Consequently,

$$
\frac{d}{d r} \mathrm{~A}_{\alpha, \beta}(f, r)=0 \quad \forall \quad r \in\left[r_{1}, r_{2}\right]
$$

and so

$$
\int_{0}^{r}\left[\frac{d}{d t} \Phi_{A, \beta}(f, t)\right] v_{\alpha}(t) d t=0 \quad \forall \quad r \in\left[r_{1}, r_{2}\right]
$$

Then we must have

$$
\frac{d}{d t} \Phi_{A, \beta}(f, t)=0 \quad \forall \quad t \in(0, r) \quad \text { with } \quad r \in\left[r_{1}, r_{2}\right],
$$

whence getting that if $\beta<1$ then $f$ must be constant or if $\beta=1$ then $f$ must be linear, thanks to the argument for the strictness in Lemma2 (i).

It remains to check the rest of Theorem 1 .

(i) The monotonic growth of $\mathrm{A}_{\alpha, \beta}(f, \cdot)$ ensures the existence of the limit. An application of L'Hôpital's rule gives

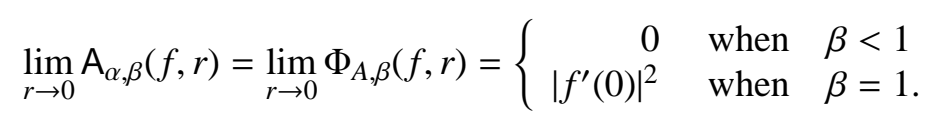

(ii) Again, the above monotonicity formula of $\mathrm{A}_{\alpha, \beta}(f, \cdot)$ plus the given condition yields that for $s \in(0,1)$,

$$
\sup _{r \in(0, s)} \mathrm{A}_{\alpha, \beta}(f, r)=\mathrm{A}_{\alpha, \beta}(f, s)<\infty
$$


Integrating by parts twice and using the monotonicity of $\Phi_{A, \beta}(f, \cdot)$, we obtain that under $0<r<s<1$,

$$
\begin{aligned}
0 & \leq \mathrm{A}_{\alpha, \beta}(f, s)-\mathrm{A}_{\alpha, \beta}(f, r) \\
& =\int_{r}^{s} \frac{d}{d t} \mathrm{~A}_{\alpha, \beta}(f, t) d t \\
& =\int_{r}^{s}\left(\int_{0}^{t}\left[\frac{d}{d \tau} \Phi_{A, \beta}(f, \tau)\right] v_{\alpha}(\tau) d \tau\right)\left[\frac{d v_{\alpha}(t)}{v_{\alpha}(t)^{2}}\right] \\
& =\int_{r}^{s}\left(v_{\alpha}(t) \Phi_{A, \beta}(f, t)-\int_{0}^{t} \Phi_{A, \beta}(f, \tau) d v_{\alpha}(\tau)\right)\left[\frac{d v_{\alpha}(t)}{v_{\alpha}(t)^{2}}\right] \\
& \leq\left[\Phi_{A, \beta}(f, s)-\Phi_{A, \beta}(f, 0)\right] \int_{r}^{s} \frac{d v_{\alpha}(t)}{v_{\alpha}(t)} .
\end{aligned}
$$

This gives the desired inequality right away. Furthermore, the above argument plus Lemma2 (i) derives the equality case.

As an immediate consequence of Theorem 11 we get a sort of "norm" estimate associated with $\Phi_{A, \beta}(f, \cdot)$.

Corollary 1. Let $-\infty<\alpha<\infty, 0 \leq \beta \leq 1$, and $f \in H(\mathbb{D})$.

(i) If $-\infty<\alpha \leq-1$, then

$$
\int_{0}^{1} \Phi_{A, \beta}(f, t) d \mu_{\alpha}(t)=\sup _{r \in(0,1)} \int_{0}^{r} \Phi_{A, \beta}(f, t) d \mu_{\alpha}(t)<\infty
$$

if and only if $f$ is constant. Moreover, $\sup _{r \in(0,1)} \mathrm{A}_{\alpha, \beta}(f, r)=\Phi_{A, \beta}(f, 1)$.

(ii) If $-1<\alpha<\infty$, then

$$
\mathrm{A}_{\alpha, \beta}(f, r) \leq \mathrm{A}_{\alpha, \beta}(f, 1):=\sup _{s \in(0,1)} \mathrm{A}_{\alpha, \beta}(f, s) \quad \forall \quad r \in(0,1),
$$

where the inequality becomes an equality for all $r \in(0,1)$ if and only if

$$
f=\left\{\begin{array}{r}
\text { constant } \text { when } \quad \beta<1 \\
\text { linear map } \quad \text { when } \quad \beta=1 .
\end{array}\right.
$$

(iii) The following function $\alpha \mapsto \mathrm{A}_{\alpha, \beta}(f, 1)$ is strictly decreasing on $(-1, \infty)$ unless

$$
f=\left\{\begin{aligned}
\text { constant } & \text { when } \quad \beta<1 \\
\text { linear map } & \text { when } \quad \beta=1 .
\end{aligned}\right.
$$

Proof. (i) By Theorem 1, we have

$$
\mathrm{A}_{\alpha, \beta}(f, r) \leq \frac{\int_{0}^{s} \Phi_{A, \beta}(f, t) d \mu_{\alpha}(t)}{v_{\alpha}(s)} \quad \forall \quad r \in(0, s) .
$$

Note that

$$
\lim _{s \rightarrow 1} v_{\alpha}(s)=\infty \quad \& \quad \lim _{s \rightarrow 1} \int_{0}^{s} \Phi_{A, \beta}(f, t) d \mu_{\alpha}(t)=\int_{0}^{1} \Phi_{A, \beta}(f, t) d \mu_{\alpha}(t) .
$$

So, the last integral is finite if and only if

$$
\Phi_{A, \beta}(f, r)=0 \quad \forall \quad r \in(0,1),
$$


equivalently, $A(f, r)=0$ holds for all $r \in(0,1)$, i.e., $f$ is constant.

For the remaining part of (i), we may assume that $f$ is not a constant map. Due to $\lim _{r \rightarrow 1} v_{\alpha}(r)=\infty$, we obtain

$$
\lim _{r \rightarrow 1} \int_{0}^{r} \Phi_{A, \beta}(f, t) d \mu_{\alpha}(t)=\int_{0}^{1} \Phi_{A, \beta}(f, t) d \mu_{\alpha}(t)=\infty .
$$

So, an application of L'Hôpital's rule yields

$$
\sup _{0<r<1} \mathrm{~A}_{\alpha, \beta}(f, r)=\lim _{r \rightarrow 1} \frac{\int_{0}^{r} \Phi_{A, \beta}(f, t) d \mu_{\alpha}(t)}{v_{\alpha}(r)}=\lim _{r \rightarrow 1} \frac{\Phi_{A, \beta}(f, r) r\left(1-r^{2}\right)^{\alpha}}{r\left(1-r^{2}\right)^{\alpha}}=\Phi_{A, \beta}(f, 1) .
$$

(ii) Under $-1<\alpha<\infty$, we have

$$
\lim _{r \rightarrow 1} v_{\alpha}(r)=v_{\alpha}(1) \quad \& \quad \lim _{r \rightarrow 1} \int_{0}^{r} \Phi_{A, \beta}(f, t) d \mu_{\alpha}(t)=\int_{0}^{1} \Phi_{A, \beta}(f, t) d \mu_{\alpha}(t) .
$$

Thus, by Theorem 1 it follows that for $r \in(0,1)$,

$$
\mathrm{A}_{\alpha, \beta}(f, r) \leq \lim _{s \rightarrow 1} \mathrm{~A}_{\alpha, \beta}(f, s)=\left[v_{\alpha}(1)\right]^{-1} \int_{0}^{1} \Phi_{A, \beta}(f, t) d \mu_{\alpha}(t)=\sup _{s \in(0,1)} \mathrm{A}_{\alpha, \beta}(f, s) .
$$

The equality case just follows from a straightforward computation and Theorem 1

(iii) Suppose $-1<\alpha_{1}<\alpha_{2}<\infty$ and $\mathrm{A}_{\alpha_{1}, \beta}(f, 1)<\infty$, then integrating by parts twice, we obtain

$$
\begin{aligned}
\mathrm{A}_{\alpha_{2}, \beta}(f, 1) & =\left[v_{\alpha_{2}}(1)\right]^{-1} \int_{0}^{1} \Phi_{A, \beta}(f, r) d \mu_{\alpha_{2}}(r) \\
& =\left[v_{\alpha_{2}}(1)\right]^{-1} \int_{0}^{1}\left(1-r^{2}\right)^{\alpha_{2}-\alpha_{1}} \frac{d}{d r}\left[\int_{0}^{r} \Phi_{A, \beta}(f, t) d \mu_{\alpha_{1}}(t)\right] d r \\
& =\left[v_{\alpha_{2}}(1)\right]^{-1}\left[-\int_{0}^{1}\left(\int_{0}^{r} \Phi_{A, \beta}(f, t) d \mu_{\alpha_{1}}(t)\right) d\left(1-r^{2}\right)^{\alpha_{2}-\alpha_{1}}\right] \\
& \leq\left[v_{\alpha_{2}}(1)\right]^{-1} \mathrm{~A}_{\alpha_{1}, \beta}(f, 1) \int_{0}^{1} v_{\alpha_{1}}(r) d\left[-\left(1-r^{2}\right)^{\alpha_{2}-\alpha_{1}}\right] \\
& =\mathrm{A}_{\alpha_{1}, \beta}(f, 1)\left[v_{\alpha_{2}}(1)\right]^{-1}\left[\int_{0}^{1}\left(1-r^{2}\right)^{\alpha_{2}-\alpha_{1}} d \mu_{\alpha_{1}}(r)\right] \\
& =\mathrm{A}_{\alpha_{1}, \beta}(f, 1),
\end{aligned}
$$

thereby establishing $\mathbf{A}_{\alpha_{2}, \beta}(f, 1) \leq \mathbf{A}_{\alpha_{1}, \beta}(f, 1)$. If this last inequality becomes equality, then the above argument forces

$$
\int_{0}^{r} \Phi_{A, \beta}(f, t) d \mu_{\alpha_{1}}(t)=\mathrm{A}_{\alpha_{1}, \beta}(f, 1) v_{\alpha_{1}}(r) \quad \forall \quad r \in(0,1),
$$

whence yielding (via the just-verified (ii))

$$
f=\left\{\begin{aligned}
\text { constant } & \text { when } \quad \beta<1 \\
\text { linear map } & \text { when } \quad \beta=1 .
\end{aligned}\right.
$$


2.3. Monotonic Growth of $\mathrm{L}_{\alpha, \beta}(f, \cdot)$. Correspondingly, we first have the following Schwarz type lemma.

Proposition 2. Let $-\infty<\alpha<\infty, 0 \leq \beta \leq 1$, and $f \in H(\mathbb{D})$ be of the form $f(z)=a_{0}+\sum_{k=n}^{\infty} a_{k} z^{k}$ with $n \in \mathbb{N}$. Then

$$
(2 \pi)^{1-\beta}\left[\frac{\left|f^{(n)}(0)\right|}{n !}\right] \leq \mathrm{L}_{\alpha, \beta}(f, r)\left[\frac{v_{\alpha}(r)}{\int_{0}^{r} t^{n-\beta} d \mu_{\alpha}(t)}\right] \quad \forall \quad r \in(0,1)
$$

with equality when and only when $f=a_{0}+a_{n} z^{n}$.

Proof. This follows from Lemma1(ii) and its equality case.

The coming-up-next monotonicity contains a hypothesis stronger than that for Theorem 1

Theorem 2. Let $-\infty<\alpha<\infty, 0 \leq \beta \leq 1$, and $f \in U(\mathbb{D})$ or $f(z)=a_{0}+a_{n} z^{n}$ with $n \in \mathbb{N}$. Then $r \mapsto \mathrm{L}_{\alpha, \beta}(f, r)$ is strictly increasing on $(0,1)$ unless

$$
f=\left\{\begin{aligned}
\text { constant } & \text { when } \quad \beta<1 \\
\text { linear map } & \text { when } \quad \beta=1 .
\end{aligned}\right.
$$

Consequently,

(i)

(ii) If

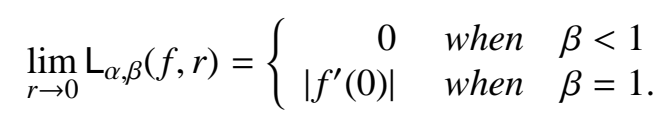

$$
\Phi_{L, \beta}(f, 0):=\lim _{r \rightarrow 0} \Phi_{L, \beta}(f, r) \quad \& \quad \Phi_{L, \beta}(f, 1):=\lim _{r \rightarrow 1} \Phi_{L, \beta}(f, r)<\infty,
$$

then

$$
0<r<s<1 \Rightarrow 0 \leq \frac{\mathrm{L}_{\alpha, \beta}(f, s)-\mathrm{L}_{\alpha, \beta}(f, r)}{\log v_{\alpha}(s)-\log v_{\alpha}(r)} \leq \Phi_{L, \beta}(f, s)-\Phi_{L, \beta}(f, 0)
$$

with equality if and only if

$$
f=\left\{\begin{aligned}
\text { constant } & \text { when } \quad \beta<1 \\
\text { linear map } & \text { when } \quad \beta=1 .
\end{aligned}\right.
$$

In particular, $t \mapsto \mathrm{L}_{\alpha, \beta}(f, t)$ is Lipschitz with respect to $\log v_{\alpha}(t)$ for $t \in(0,1)$.

Proof. Similar to that for Theorem11 but this time by Lemma2(ii).

Naturally, we can establish the so-called "norm" estimate associated to $\Phi_{L, \beta}(f, \cdot)$.

Corollary 2. Let $0 \leq \beta \leq 1$ and $f \in U(\mathbb{D})$ or $f(z)=a_{0}+a_{n} z^{n}$ with $n \in \mathbb{N}$.

(i) If $-\infty<\alpha \leq-1$, then

$$
\int_{0}^{1} \Phi_{L, \beta}(f, t) d \mu_{\alpha}(t)=\sup _{r \in(0,1)} \int_{0}^{r} \Phi_{L, \beta}(f, t) d \mu_{\alpha}(t)<\infty
$$

if and only if $f$ is constant. Moreover, $\sup _{r \in(0,1)} \mathrm{L}_{\alpha, \beta}(f, r)=\Phi_{L, \beta}(f, 1)$. 
(ii) If $-1<\alpha<\infty$, then

$$
\mathrm{L}_{\alpha, \beta}(f, r) \leq \mathrm{L}_{\alpha, \beta}(f, 1):=\sup _{s \in(0,1)} \mathrm{L}_{\alpha, \beta}(f, s) \quad \forall \quad r \in(0,1),
$$

where the inequality becomes an equality for all $r \in(0,1)$ if and only if

$$
f=\left\{\begin{aligned}
\text { constant } & \text { when } \quad \beta<1 \\
\text { linear map } & \text { when } \quad \beta=1 .
\end{aligned}\right.
$$

(iii) $\alpha \mapsto \mathrm{L}_{\alpha, \beta}(f, 1)$ is strictly decreasing on $(-1, \infty)$ unless

$$
f=\left\{\begin{aligned}
\text { constant } & \text { when } \quad \beta<1 \\
\text { linear map } & \text { when } \quad \beta=1 .
\end{aligned}\right.
$$

Proof. The argument is similar to that for Corollary 1 , but via Lemma2(ii).

\section{LOGARITHMIC CONVEXITY}

In this section, we treat the convexities of the functions: $\log r \mapsto \log \mathrm{A}_{\alpha, \beta}(f, r)$ and $\log r \mapsto \log \mathrm{L}_{\alpha, \beta}(f, r)$ for $r \in(0,1)$.

3.1. Two More Lemmas. The following are two technical preliminaries.

Lemma 3. [10, Corollaries 2-3 \& Proposition 7] Suppose $f(x)$ and $\left\{h_{k}(x)\right\}_{k=0}^{\infty}$ are positive and twice differentiable for $x \in(0,1)$ such that the function $H(x)=$ $\sum_{k=0}^{\infty} h_{k}(x)$ is also twice differentiable for $x \in(0,1)$. Then:

(i) $\log x \mapsto \log f(x)$ is convex if and only if $\log x \mapsto \log f\left(x^{2}\right)$ is convex.

(ii) The function $\log x \mapsto \log f(x)$ is convex if and only if the D-notation of $f$

$$
D(f(x)):=\frac{f^{\prime}(x)}{f(x)}+x\left(\frac{f^{\prime}(x)}{f(x)}\right)^{\prime} \geq 0 \quad \forall \quad x \in(0,1) .
$$

(iii) If for each $k$ the function $\log x \mapsto \log h_{k}(x)$ is convex, then $\log x \mapsto \log H(x)$ is also convex.

Lemma 4. Let $f \in H(\mathbb{D})$. Then $f$ belongs to $U(\mathbb{D})$ provided that one of the following two conditions is valid:

(i) [8] or [1, Lemma 2.1]

$$
f(0)=f^{\prime}(0)-1=0 \quad \& \quad\left|\frac{z^{2} f^{\prime}(z)}{f^{2}(z)}-1\right|<1 \quad \forall \quad z \in \mathbb{D} .
$$

(ii) [7, Theorem 1] or [4, Theorem 8.12]

$$
\left|\left[\frac{f^{\prime \prime}(z)}{f^{\prime}(z)}\right]^{\prime}-\frac{1}{2}\left[\frac{f^{\prime \prime}(z)}{f^{\prime}(z)}\right]^{2}\right| \leq 2\left(1-|z|^{2}\right)^{-2} \quad \forall \quad z \in \mathbb{D} .
$$


3.2. Log-convexity for $\mathbf{A}_{\alpha, \beta}(f, \cdot)$. Such a property is given below.

Theorem 3. Let $0 \leq \beta \leq 1$ and $0<r<1$.

(i) If $\alpha \in(-\infty,-3)$, then there exist $f, g \in H(\mathbb{D})$ such that $\log r \mapsto \log \mathrm{A}_{\alpha, \beta}(f, r)$ is not convex and $\log r \mapsto \log \mathrm{A}_{\alpha, \beta}(g, r)$ is not concave.

(ii) If $\alpha \in[-3,0]$, then $\log r \mapsto \log \mathrm{A}_{\alpha, 1}\left(a_{n} z^{n}, r\right)$ is convex for $a_{n} \neq 0$ with $n \in \mathbb{N}$. Consequently,

$$
\log r \mapsto \log \mathrm{A}_{\alpha, 1}(f, r)
$$

is convex for all $f \in U(\mathbb{D})$.

(iii) If $\alpha \in(0, \infty)$, then $\log r \mapsto \log \mathrm{A}_{\alpha, \beta}\left(a_{n} z^{n}, r\right)$ is not convex for $a_{n} \neq 0$ and $n \in \mathbb{N}$.

Proof. The key issue is to check whether or not $\log r \mapsto \log \mathrm{A}_{\alpha, \beta}\left(z^{n}, r\right)$ is convex for $r \in(0,1)$.

To see this, let us borrow some symbols from [10]. For $\lambda \geq 0$ and $0<x<1$ we define

$$
f_{\lambda}(x)=\int_{0}^{x} t^{\lambda}(1-t)^{\alpha} d t
$$

and

$$
\Delta(\lambda, x)=\frac{f_{\lambda}^{\prime}(x)}{f_{\lambda}(x)}+x\left(\frac{f_{\lambda}^{\prime}(x)}{f_{\lambda}(x)}\right)^{\prime}-\left[\frac{f_{0}^{\prime}(x)}{f_{0}(x)}+x\left(\frac{f_{0}^{\prime}(x)}{f_{0}(x)}\right)^{\prime}\right] .
$$

Given $n \in \mathbb{N}$. A simple calculation shows $\Phi_{A, \beta}\left(z^{n}, t\right)=\pi^{1-\beta} t^{2(n-\beta)}$, and then a change of variable derives

$$
\begin{aligned}
\mathrm{A}_{\alpha, \beta}\left(z^{n}, r\right) & =\frac{\int_{0}^{r} \Phi_{A, \beta}\left(z^{n}, t\right) d \mu_{\alpha}(t)}{v_{\alpha}(r)} \\
& =\frac{\pi^{1-\beta} \int_{0}^{r^{2}} t^{n-\beta}(1-t)^{\alpha} d t}{\int_{0}^{r^{2}}(1-t)^{\alpha} d t} \\
& =\pi^{1-\beta}\left[\frac{f_{n-\beta}\left(r^{2}\right)}{f_{0}\left(r^{2}\right)}\right] .
\end{aligned}
$$

In accordance with Lemma 3 (i)-(ii), it is readily to work out that $\log r \mapsto$ $\log \mathrm{A}_{\alpha, \beta}\left(z^{n}, r\right)$ is convex for $r \in(0,1)$ if and only if $\Delta(n-\beta, x) \geq 0$ for any $x \in(0,1)$.

(i) Under $\alpha \in(-\infty,-3)$, we follow the argument for [10, Proposition 6] to get

$$
\lim _{x \rightarrow 1} \Delta(\lambda, x)=\frac{\lambda(\alpha+1)(\lambda+2+\alpha)}{(\alpha+2)^{2}(\alpha+3)} .
$$

Choosing

$$
f(z)=z^{n}=\left\{\begin{array}{rll}
z & \text { when } & \beta<1 \\
z^{2} & \text { when } \quad \beta=1
\end{array}\right.
$$

and $\lambda=n-\beta$, we find $\lim _{x \rightarrow 1} \Delta(\lambda, x)<0$, whence deriving that $\log r \mapsto \log A_{\alpha}(f, r)$ is not convex.

In the meantime, picking $n \in \mathbb{N}$ such that $n>\beta-(2+\alpha)$ and putting $g(z)=z^{n}$, we obtain

$$
\lim _{x \rightarrow 1} \Delta(n-\beta, x)=\frac{(n-\beta)(\alpha+1)(n-\beta+2+\alpha)}{(\alpha+2)^{2}(\alpha+3)}>0,
$$


whence deriving that $\log r \mapsto \log \mathrm{A}_{\alpha, \beta}(g, r)$ is not concave.

(ii) Under $\alpha \in[-3,0]$, we handle the two situations.

Situation 1: $f \in U(\mathbb{D})$. Upon writing $f(z)=\sum_{n=0}^{\infty} a_{n} z^{n}$, we compute

$$
\Phi_{A, 1}(f(z), t)=\left(\pi t^{2}\right)^{-1} A(f, t)=\sum_{n=0}^{\infty} n\left|a_{n}\right|^{2} t^{2(n-1)},
$$

and consequently,

$$
\mathrm{A}_{\alpha, 1}(f, r)=\frac{\sum_{n=0}^{\infty} n\left|a_{n}\right|^{2} \int_{0}^{r}\left(\pi t^{2}\right)^{-1} A\left(z^{n}, t\right) d \mu_{\alpha}(t)}{v_{\alpha}(r)}=\sum_{n=0}^{\infty} n\left|a_{n}\right|^{2} \mathrm{~A}_{\alpha, 1}\left(z^{n}, r\right) .
$$

So, by Lemmaß(iii), we see that the convexity of

$$
\log r \mapsto \log \mathrm{A}_{\alpha, 1}(f, r) \quad \text { under } \quad f \in U(\mathbb{D})
$$

follows from the convexity of

$$
\log r \mapsto \log \mathrm{A}_{\alpha, 1}\left(z^{n}, r\right) \quad \text { under } \quad n \in \mathbb{N} .
$$

So, it remains to verify this last convexity via the coming-up-next consideration.

Situation 2: $f(z)=a_{n} z^{n}$ with $a_{n} \neq 0$. Three cases are required to control.

Case 1: $\alpha=0$. An easy computation shows

$$
\mathrm{A}_{0,1}\left(z^{n}, r\right)=n^{-1} r^{2(n-1)}
$$

and so $\log r \mapsto \log \mathrm{A}_{0,1}\left(z^{n}, r\right)$ is convex.

Case 2: $-2 \leq \alpha<0$. Under this condition, we see from the arguments for [10, Propositions 4-5] that

$$
\Delta(n-1, x) \geq 0 \quad \forall \quad n-1 \geq 0 \quad \& \quad 0<x<1,
$$

and so that $\log r \mapsto \log \mathrm{A}_{\alpha, 1}\left(z^{n}, r\right)$ is convex.

Case 3: $-3 \leq \alpha<-2$. With the assumption, we also get from the arguments for [10, Propositions 4-5] that

$$
\Delta(n-1, x) \geq \Delta(-2-\alpha, x)>0 \quad \forall \quad x \in(0,1) \& n-1 \in[-2-\alpha, \infty)
$$

and so that $\log r \mapsto \log \mathrm{A}_{\alpha, 1}\left(z^{n}, r\right)$ is convex when $n \geq 2$. Here it is worth noting that the convexity of $\log r \mapsto \log \mathrm{A}_{\alpha, 1}(z, r)=0$ is trivial.

(iii) Under $0<\alpha<\infty$, from the argument for [10, Proposition 6] we know that $\Delta(n-\beta, x)<0$ as $x$ is sufficiently close to 1 . Thus $\log r \mapsto \log \mathrm{A}_{\alpha, \beta}\left(a_{n} z^{n}, r\right)$ is not convex under $a_{n} \neq 0$.

The following illustrates that the function $\log r \mapsto \log \mathrm{A}_{\alpha, \beta}(f, r)$ is not always concave for $\alpha>0,0 \leq \beta \leq 1$, and $f \in U(\mathbb{D})$.

Example 1. Let $\alpha=1, \beta \in\{0,1\}$, and $f(z)=z+\frac{z^{2}}{2}$. Then the function $\log r \mapsto$ $\log \mathrm{A}_{\alpha, \beta}(f, r)$ is neither convex nor concave for $r \in(0,1)$.

Proof. A direct computation shows

$$
\left|\frac{z^{2} f^{\prime}(z)}{f^{2}(z)}-1\right|=\left|\frac{z^{2}(1+z)}{\left(z+\frac{z^{2}}{2}\right)^{2}}-1\right|=\frac{|z|^{2}}{|z+2|^{2}}<1
$$


since

$$
|z|<1<2-|z| \leq|z+2| \quad \forall \quad z \in \mathbb{D} .
$$

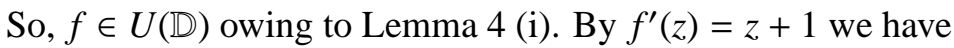

$$
A(f, t)=\int_{t \mathbb{D}}|z+1|^{2} d A(z)=\pi\left(t^{2}+\frac{t^{4}}{2}\right),
$$

plus

$$
\int_{0}^{r} \Phi_{A, \beta}(f, t) d \mu_{1}(t)=\left\{\begin{array}{rr}
\frac{\pi}{2}\left(r^{4}-\frac{r^{6}}{3}-\frac{r^{8}}{4}\right) & \text { when } \quad \beta=0 \\
r^{2}-\frac{r^{4}}{4}-\frac{r^{6}}{6} & \text { when } \quad \beta=1
\end{array}\right.
$$

Meanwhile,

$$
v_{1}(r)=\int_{0}^{r}\left(1-t^{2}\right) d t^{2}=r^{2}-\frac{r^{4}}{2}
$$

So, we get

$$
\mathrm{A}_{1, \beta}(f, r)=\left\{\begin{array}{ccc}
\frac{\pi\left(12 r^{2}-4 r^{4}-3 r^{6}\right)}{12\left(2-r^{2}\right)} & \text { when } & \beta=0 \\
\frac{12-3 r^{2}-2 r^{4}}{6\left(2-r^{2}\right)} & \text { when } \beta=1
\end{array}\right.
$$

and in turn consider the logarithmic convexities of the following function

$$
h_{\beta}(x)=\left\{\begin{array}{ccc}
\frac{12 x-4 x^{2}-3 x^{3}}{2-x} & \text { when } \beta=0 \\
\frac{12-3 x-2 x^{2}}{2-x} & \text { when } \beta=1
\end{array}\right.
$$

for $x \in(0,1)$.

Using the so-called D-notation in Lemma3, we have

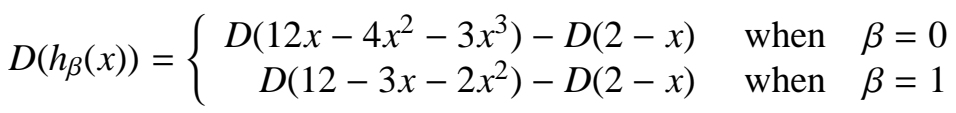

for $x \in(0,1)$. By an elementary calculation, we get

$$
\left\{\begin{array}{r}
D\left(12 x-4 x^{2}-3 x^{3}\right)=\frac{-48-144 x+12 x^{2}}{\left(12-4 x-3 x^{2}\right)^{2}} \\
D(2-x)=\frac{-2}{(2-x)^{2}} \\
D\left(12-3 x-2 x^{2}\right)=\frac{-36-96 x+6 x^{2}}{\left(12-3 x-2 x^{2}\right)^{2}}
\end{array}\right.
$$

Consequently,

$$
D\left(h_{\beta}(x)\right)=\left\{\begin{array}{lll}
\frac{2 g_{\beta}(x)}{\left(12-4 x-3 x^{2}\right)^{2}(2-x)^{2}} & \text { when } \quad \beta=0 \\
\frac{2 g_{\beta}(x)}{\left(12-3 x-2 x^{2}\right)^{2}(2-x)^{2}} & \text { when } \quad \beta=1,
\end{array}\right.
$$

where

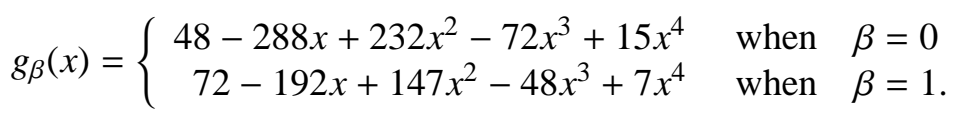

Now, under $x \in(0,1)$ we find

$$
g_{0}^{\prime}(x)=-288+464 x-216 x^{2}+60 x^{3} \quad \& \quad g_{0}^{\prime \prime}(x)=464-432 x+180 x^{2} .
$$


Clearly, $g_{0}^{\prime \prime}(x)$ is an open-upward parabola with the axis of symmetry $x=\frac{6}{5}>1$. By $g_{0}^{\prime \prime}(1)=212>0$ and the monotonicity of $g_{0}^{\prime \prime}$ on $(0,1)$, we have $g_{0}^{\prime \prime}(x)>0$ for all $x \in(0,1)$. Thus $g_{0}^{\prime}$ is increasing on $(0,1)$. The following condition

$$
g_{0}^{\prime}(0)=-288<0 \quad \& \quad g_{0}^{\prime}(1)=20>0
$$

yields an $x_{1} \in(0,1)$ such that $g_{0}^{\prime}(x)<0$ for $x \in\left(0, x_{1}\right)$ and $g_{0}^{\prime}(x)>0$ for $x \in\left(x_{1}, 1\right)$. Since $g_{0}(0)=48$ and $g_{0}(1)=-65$, there exists an $x_{0} \in(0,1)$ such that $g_{0}(x)>0$ for $x \in\left(0, x_{0}\right)$ and $g_{0}(x)<0$ for $x \in\left(x_{0}, 1\right)$. Thus the function $\log x \mapsto \log h_{0}(x)$ is neither convex nor concave.

Similarly, under $x \in(0,1)$ we have

$$
g_{1}^{\prime}(x)=-192+294 x-144 x^{2}+28 x^{3} \quad \& \quad g_{1}^{\prime \prime}(x)=294-288 x+84 x^{2} .
$$

Obviously, $g_{1}^{\prime \prime}(x)$ is an open-upward parabola with the axis of symmetry $x=\frac{12}{7}>$ 1. By $g_{1}^{\prime \prime}(1)=90>0$ and the monotonicity of $g_{1}^{\prime \prime}$ on $(0,1)$, we have $g_{1}^{\prime \prime}(x)>0$ for all $x \in(0,1)$. Thus $g_{1}^{\prime}$ is increasing on $(0,1)$. The following condition

$$
g_{1}^{\prime}(0)=-192<0 \quad \& \quad g_{1}^{\prime}(1)=-14<0
$$

yields $g_{1}^{\prime}(x)<0$ for $x \in(0,1)$. Since $g_{1}(0)=72$ and $g_{1}(1)=-14$, there exists an $x_{0} \in(0,1)$ such that $g_{1}(x)>0$ for $x \in\left(0, x_{0}\right)$ and $g_{1}(x)<0$ for $x \in\left(x_{0}, 1\right)$. Thus the function $\log x \mapsto \log h_{1}(x)$ is neither convex nor concave.

3.3. Log-convexity for $\mathrm{L}_{\alpha, \beta}(f, \cdot)$. Analogously, we can establish the expected convexity for the mixed lengths.

Theorem 4. Let $0 \leq \beta \leq 1$ and $0<r<1$.

(i) If $\alpha \in(-\infty,-3)$, then there exist $f, g \in H(\mathbb{D})$ such that $\log r \mapsto \log \mathrm{L}_{\alpha, \beta}(f, r)$ is not convex and $\log r \mapsto \log \mathrm{L}_{\alpha, \beta}(g, r)$ is not concave.

(ii) If $\alpha \in[-3,0]$, then $\log r \mapsto \log \mathrm{L}_{\alpha, 1}\left(a_{n} z^{n}, r\right)$ is convex for $a_{n} \neq 0$ with $n \in \mathbb{N}$. Consequently, $\log r \mapsto \log \mathrm{L}_{\alpha, 1}(f, r)$ is convex for $f \in U(\mathbb{D})$.

(iii) If $\alpha \in(0, \infty)$, then $\log r \mapsto \log \mathrm{L}_{\alpha, \beta}\left(a_{n} z^{n}, r\right)$ is not convex for $a_{n} \neq 0$ and $n \in \mathbb{N}$.

Proof. The argument is similar to that for Theorem 3 except using the following statement for $\alpha \in[-3,0]$ - If $f \in U(\mathbb{D})$, then there exists $g(z)=\sum_{n=0}^{\infty} b_{n} z^{n}$ such that $g$ is the square root of the zero-free derivative $f^{\prime}$ on $\mathbb{D}$ and $f^{\prime}(0)=g^{2}(0)$, and hence

$$
\begin{aligned}
\Phi_{L, 1}(f, t) & =(2 \pi t)^{-1} \int_{t \mathbb{T}}\left|f^{\prime}(z)\right||d z| \\
& =(2 \pi t)^{-1} \int_{t \mathbb{T}}|g(z)|^{2}|d z| \\
& =\sum_{n=0}^{\infty}\left|b_{n}\right|^{2} t^{2 n} .
\end{aligned}
$$

Our concluding example shows that under $0<\alpha<\infty$ and $0 \leq \beta \leq 1$ one cannot get that $\log \mathrm{L}_{\alpha, \beta}(f, r)$ is convex or concave in $\log r$ for all functions $f \in U(\mathbb{D})$. 
Example 2. Let $\alpha=1, \beta \in\{0,1\}$, and $f(z)=(z+2)^{3}$. Then the function $\log r \mapsto$ $\log \mathrm{L}_{\alpha, \beta}(f, r)$ is neither convex nor concave for $r \in(0,1)$.

Proof. Clearly, we have

$$
f^{\prime}(z)=3(z+2)^{2} \& f^{\prime \prime}(z)=6(z+2)
$$

as well as the Schwarizian derivative

$$
\left[\frac{f^{\prime \prime}(z)}{f^{\prime}(z)}\right]^{\prime}-\frac{1}{2}\left[\frac{f^{\prime \prime}(z)}{f^{\prime}(z)}\right]^{2}=\frac{-4}{(z+2)^{2}}
$$

It is easy to see that

$$
\sqrt{2}\left(1-|z|^{2}\right) \leq 2-|z| \quad \forall \quad z \in \mathbb{D} \text {. }
$$

So,

$$
\left|\left[\frac{f^{\prime \prime}(z)}{f^{\prime}(z)}\right]^{\prime}-\frac{1}{2}\left[\frac{f^{\prime \prime}(z)}{f^{\prime}(z)}\right]^{2}\right|=\frac{4}{|z+2|^{2}} \leq \frac{4}{(2-|z|)^{2}} \leq \frac{2}{\left(1-|z|^{2}\right)^{2}} .
$$

By Lemma 4 (ii), $f$ belongs to $U(\mathbb{D})$. Consequently,

$$
L(f, t)=\int_{0}^{2 \pi}\left|f^{\prime}\left(t e^{i \theta}\right)\right| t d \theta=6 \pi t\left(t^{2}+4\right)
$$

and

$$
\int_{0}^{r} \Phi_{L, \beta}(f, t) d \mu_{1}(t)=\left\{\begin{aligned}
12 \pi\left(\frac{4}{3} r^{3}-\frac{3}{5} r^{5}-\frac{1}{7} r^{7}\right) & \text { when } \quad \beta=0 \\
12 r^{2}-\frac{9}{2} r^{4}-r^{6} & \text { when } \quad \beta=1 .
\end{aligned}\right.
$$

Note that $v_{1}(r)=r^{2}-\frac{r^{4}}{2}$. So,

$$
\mathrm{L}_{1, \beta}(f, r)=\left\{\begin{aligned}
\frac{24 \pi\left(140 r-63 r^{3}-15 r^{5}\right)}{105\left(2-r^{2}\right)} & \text { when } \quad \beta=0 \\
\frac{24-9 r^{2}-2 r^{4}}{2-r^{2}} & \text { when } \beta=1 .
\end{aligned}\right.
$$

To gain our conclusion, we only need to consider the logarithmic convexity of the function

$$
h_{\beta}(x)=\left\{\begin{array}{rll}
\frac{140 x-63 x^{3}-15 x^{5}}{2-x^{2}} & \text { when } \beta=0 \\
\frac{24-9 x-2 x^{2}}{2-x} & \text { when } \beta=1 .
\end{array}\right.
$$

Case 1: $\beta=0$. Applying the definition of $D$-notation, we obtain

$$
D\left(140 x-63 x^{3}-15 x^{5}\right)=\frac{-35280 x-33600 x^{3}+3780 x^{5}}{\left(140-63 x^{2}-15 x^{4}\right)^{2}}
$$

and

whence reaching

$$
D\left(2-x^{2}\right)=\frac{-8 x}{\left(2-x^{2}\right)^{2}}
$$

$D\left(h_{0}(x)\right)=D\left(140 x-63 x^{3}-15 x^{5}\right)-D\left(2-x^{2}\right)=\frac{4 x g_{0}(x)}{\left(140-63 x^{2}-15 x^{4}\right)^{2}\left(2-x^{2}\right)^{2}}$,

where

$$
g_{0}(x)=3920-33600 x^{2}+28098 x^{4}-8400 x^{6}+1395 x^{8} .
$$


Obviously,

$$
g_{0}(0)=3920>0 \quad \& \quad g_{0}(1)=-8587<0 .
$$

Now letting $s=x^{2}$, we get

$$
g_{0}(x)=G_{0}(s)=3920-33600 s+28098 s^{2}-8400 s^{3}+1395 s^{4},
$$

and

$G_{0}^{\prime}(s)=-33600+56196 s-25200 s^{2}+5580 s^{3} \& G_{0}^{\prime \prime}(s)=56196-50400 s+16740 s^{2}$.

Since the axis of symmetry of $G_{0}^{\prime \prime}$ is $s=\frac{140}{93}>1, G_{0}^{\prime \prime}$ is decreasing on $(0,1)$. Due to $G_{0}^{\prime \prime}(1)=22536>0$, we have $G_{0}^{\prime \prime}(s)>0$ for all $s \in(0,1)$, i.e., $G_{0}^{\prime}(s)$ is increasing on $(0,1)$. By

$$
G_{0}^{\prime}(0)=-33600<0 \quad \& \quad G_{0}^{\prime}(1)=2976>0,
$$

we conclude that there exists an $s_{0} \in(0,1)$ such that $G_{0}^{\prime}(s)<0$ for $s \in\left(0, s_{0}\right)$ and $G_{0}^{\prime}(s)>0$ for $s \in\left(s_{0}, 1\right)$. Then there exists an $x_{0} \in(0,1)$ such that $g_{0}(x)$ is decreasing for $x \in\left(0, x_{0}\right)$ and $g_{0}(x)$ is increasing for $x \in\left(x_{0}, 1\right)$. Thus there exists an $x_{1} \in(0,1)$ such that $g_{0}(x)>0$ for $x \in\left(0, x_{1}\right)$ and $g_{0}(x)<0$ for $x \in\left(x_{1}, 1\right)$. As a result, we find that $\log r \mapsto \log \mathrm{L}_{\alpha, 0}(f, r)$ is neither concave nor convex.

Case 2: $\beta=1$. Again using the $D$-notation, we obtain

$$
D\left(24-9 x-2 x^{2}\right)=\frac{-216-192 x+18 x^{2}}{\left(24-9 x-2 x^{2}\right)^{2}}
$$

and

$$
D(2-x)=\frac{-2}{(2-x)^{2}}
$$

whence deriving

$$
D\left(h_{1}(x)\right)=D\left(24-9 x-2 x^{2}\right)-D(2-x)=\frac{2 g_{1}(x)}{\left(24-9 x-2 x^{2}\right)^{2}(2-x)^{2}},
$$

where

$$
g_{1}(x)=144-384 x+297 x^{2}-96 x^{3}+13 x^{4} .
$$

Now we have

$$
g_{1}^{\prime}(x)=-384+594 x-288 x^{2}+52 x^{3} \quad \& \quad g_{1}^{\prime \prime}(x)=594-576 x+156 x^{2} .
$$

Since the axis of symmetry of $g_{1}^{\prime \prime}(x)$ is $x=\frac{24}{13}>1, g_{1}^{\prime \prime}(x)$ is decreasing on $(0,1)$. Due to $g_{1}^{\prime \prime}(1)=174>0$, we have $g_{1}^{\prime \prime}(x)>0$ for all $x \in(0,1)$, i.e., $g_{1}^{\prime}(x)$ is increasing on $(0,1)$. By

$$
g_{1}^{\prime}(0)=-384<0 \quad \& \quad g_{1}^{\prime}(1)=-26<0,
$$

we conclude that $g_{1}^{\prime}(x)<0$ for $x \in(0,1)$. Obviously,

$$
g_{1}(0)=144>0 \quad \& \quad g_{1}(1)=-26<0 .
$$

Hence there exists an $x_{0} \in(0,1)$ such that $g_{1}(x)>0$ for $x \in\left(0, x_{0}\right)$ and $g_{1}(x)<0$ for $x \in\left(x_{0}, 1\right)$. Consequently, we find that $\log r \mapsto \log \mathrm{L}_{\alpha, \beta=1}(f, r)$ is neither concave nor convex. 


\section{REFERENCES}

[1] M. H. Al-Abbadi and M. Darus, Angular estimates for certain analytic univalent functions, Int. J. Open Problems Complex Analysis 2 (2010), 212-220.

[2] J. Arazy, S. D. Fisher and J. Peetre, Möbius invariant function spaces, J. Reine Angew. Math. 363 (1985), 110-145.

[3] R. B. Burckel, D. E. Marshall, D. Minda, P. Poggi-Corradini and T. J. Ransford, Area, capacity and diameter versions of Schwarz's Lemma, Conform. Geom. Dyn. 12 (2008), 133-152.

[4] P. L. Duren, Univalent functions, Springer-verlag, NewYork, 1983.

[5] P. Järvi, On some function-theoretic extremal problems, Complex Variables Theory Appl. 24 (1994), 267-270.

[6] T. H. Macgregor, Length and area estimates for analytic functions, Michigan Mathe. J. 11 (1964), 317-320.

[7] Z. Nehari, The Schwarzian derivative and schlicht functions, Bull. Amer. Math. Soc. 55 (1949), $545-551$

[8] M. Nunokawa, On some angular estimates of analytic functions, Math. Japon. 41 (1995), no. 2, 447-452.

[9] G. Pólya and G. Szegö, Problems and Theorems in Analysis, I., Springer 1978.

[10] C. Wang and K. Zhu, Logarithmic convexity of area integral means for analytic functions, arXiv:1101.2998 1 [math.CV]15 Jan 2011.

[11] J. Xiao and K. Zhu, Volume integral means of holomorphic functions, Proc. Amer. Math. Soc. 139 (2011), 1455-1465.

[12] S. Yamashita, Length estimates for holomorphic functions, Proc. Amer. Math. Soc. 81 (1981), 250-252.

Department of Mathematics and Statistics, Memorial University, NL A1C 5S7, Canada

E-mail address: jxiao@mun.ca; wenxupine@gmail.com 